\title{
Load Distribution Analysis and Experimental Verification of TBM Thread Connection Structure
}

\author{
Xiaodong Nie ${ }^{1, ~ a}$, Ailian $\mathrm{Hu}^{1, \mathrm{~b}}$, Junzhou $\mathrm{Huo}^{2, c, ~}{ }^{*}$ and Jinchi Guo ${ }^{2, \mathrm{~d}}$ \\ ${ }^{1}$ Ergis River Basin Development Project Construction Management Bureau, Xinjiang Urumchi 830000, China \\ ${ }^{2}$ School of Mechanical Engineering, Dalian University of Technology, Liaoning Dalian 116024, China \\ a 412498986@qq.com, b957139099@qq.com, c239022563@qq.com, ${ }^{\mathrm{d}}$ 289947122@qq.com
}

Keywords: TBM, thread, connection structure, load distribution.

\begin{abstract}
In order to analyze the fracture of bolts at the flange of TBM cutterhead, the load distribution of the thread in the standard thread connection structure was studied. Based on theoretical analysis and Finite element simulation analysis, starting from the connection structure of bolt, the load distribution of a thread connection structure under axial load was studied, and the maximum stress at the root of each whorl was obtained by Abaqus simulation. Finally, the fatigue test of double threaded studs with standard thread connection structure was carried out. The load distribution law of each circle screw thread was verified through a set of stud fatigue fracture position. Found that the bolt fracture problem was not due to the excessive load on the flange, but due to the nouniform distribution of the axial force of the thread.
\end{abstract}

\section{Introduction}

The prediction theory of thread connection load distribution is first proposed by British Scientist Thorp Weiss (Sopwith) in 1940, which is the earliest theory of thread connection. In the load distribution prediction theory, the change of load is characterized by the deformation of the connector in the direction of stud shaft. In 1980, the Japanese scientist Yamamoto proposed that the deformation of thread is caused by the radial load and it's deformation. In this theory, the deformation of the threaded region is assumed to be a plane strain. Based on this theory, the axial load distribution of the bolt connection can be calculated. More research has been carried out on the calculation of screw load in domestic research. Xia Weiming [1] proposed to simulate thread connection in the finite element software. First, the shape feature of thread was ignored. Then thread connection was simulated by the contact mode between threads, and the load ratio of each threaded teeth under tension condition was obtained through simulation. Xie Liyang [2] extracted the screw connection into a elastic mechanical model through the finite element composite structure method to solve complex elastic contact problem. This model mainly analyzed the thread connection caused by external machining, assembly error and so on. Based on this method, the ideal thread load distribution was obtained.

In recent years, the finite element analysis method has been developed rapidly and has been adopted by many scholars. Shi Xiuyong [3,4], Fang Dong [5] et al analyzed the different feature part models by the finite element method, for the problem of stress concentration at the root of threaded connection. In the calculation of deformation in the connection surface and limited slip, Sun Yujuan [7] established the model on the basis of the method of symmetry, and the small sliding contact form was studied, the axial force distribution of each circle on the thread. By analyzing the influence of the number of finite element mesh on the stress and axial load distribution of bolt connection, the number of mesh was not related to axial load, but it had great influence on the distribution law of load stress. The more dense stress calculation results of the finite element division were more in accord with the experimental results. On the basis of simulation and experiment, Kim J [8] adopt four different ways of simulating bolt connection. It was found that finite element solid element could transfer contact pressure between threads. 
To sum up, in the related research of thread connection, scholars at home and abroad mainly focused on the study of internal load distribution, and because of the difficult measurement of internal load, the finite element analysis method is widely used. In addition to the simulation, this paper also uses theoretical analysis and experimental means to verify the simulation results.

\section{Load distribution in thread connection structure}

\subsection{Elastic deformation of bolted rod and nut body}

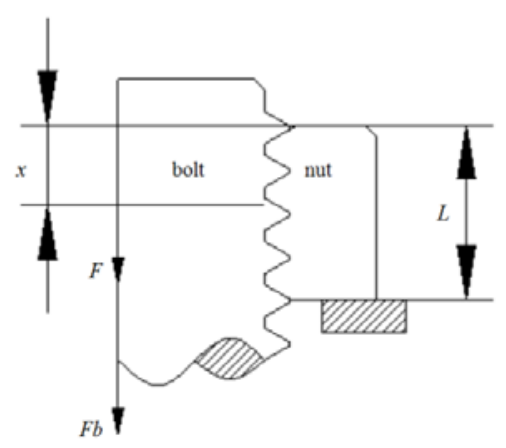

Fig. 1 The axial force of the bolt

Figure 1 is a diagram of the thread bearing the axial force. The axis of the bolt is the coordinate axis, and the center of the axis and the end of bolt is the origin of the coordinate, and the direction of the axial force is positive. The axial load on bolts is expressed by $F_{b}$. The width of the nut is expressed by $L$. The axial load of bolts from the top of thread to any position $\mathrm{x}$ is expressed by $F$, which is a function related to $x$ by calculation. The axial load of the bolts at the tip of the screw thread to the $X$ at any position is expressed by $F$. There is,

$$
F_{(x)}=\int_{0}^{x} q(x) d x
$$

where, $q(x)$ is the unit axial force of the bolt at the top of the thread to any position $x$. Bolt elongation at $\mathrm{x}-\varepsilon_{b}(x)$ and nut shortening $-\varepsilon_{n}(x)$ are as follows:

$$
\begin{gathered}
\varepsilon_{b}(x)=\int_{0}^{x} \frac{F(x)}{A_{b} \cdot E_{b}} d x \\
\varepsilon_{n}(x)=\int_{0}^{x} \frac{F(x)}{A_{n} \cdot E_{n}} d x
\end{gathered}
$$

where, $A_{b}$-Vertical section area of the bolt, $A_{n}-$ Vertical section area of nut, $E_{b}$-Elastic modulus of bolt material, $E_{n}$-Elastic modulus of nut material.

\subsection{Elastic deformation of threaded teeth}

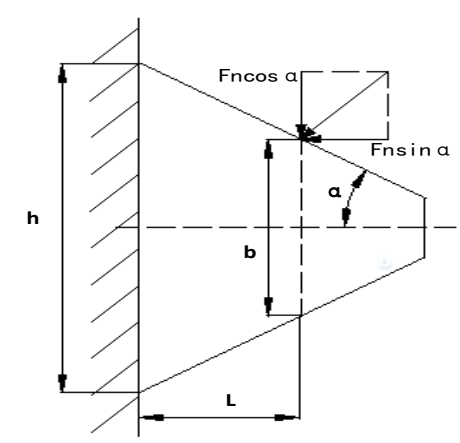

Fig.2 The force on the threaded tooth

The main factor of thread deformation is the deformation of the thread teeth under force action. 
Figure 2 is a force schematic diagram of the thread. The $b$ is length of the middle diameter of a single thread tooth, and $\mathrm{h}$ is length single thread tooth. $\mathrm{L}$ is distance between the middle diameter and the bottom of thread. The a is semiangle of thread profile. When a Fn sized force is at L distance from the bottom of thread, the deformation of the threaded teeth resulting from the axial force mainly includes the deformation caused by the bending of the thread teeth $\delta_{1}$, the deformation caused by radial force $\delta_{2}$, and the deformation caused by the root inclination of the tooth $\delta_{3}$, and the deformation caused by the shear at the root of the tooth. The deformation of the entire thread tooth is calculated [9]. Among them,

$$
\begin{gathered}
\delta_{1}=\frac{3 F_{n} \cos \alpha}{4 E}\left\{\left[1-\left(2-\frac{b}{h}\right)+2 \log _{e}\left(\frac{h}{b}\right)\right] \cot ^{3} \alpha-4\left(\frac{l}{h}\right)^{2} \tan \alpha\right\} \\
\delta_{2}=\frac{6 F_{n} \cos \alpha}{5 E} \cot \alpha \log _{e}\left(\frac{h}{b}\right) \\
\delta_{3}=\frac{12 l}{\pi E h^{2}} F_{n} \cos \alpha\left(l-\frac{h}{2}+l \tan \alpha\right) \\
\delta_{4}=\frac{2 F_{n} \cos \alpha}{\pi E}\left[\frac{P}{h} \log _{e} \frac{P+h / 2}{P-h / 2}+\frac{1}{2} \log _{e}\left(4 \frac{P^{2}}{h^{2}}-1\right)\right]
\end{gathered}
$$

For IOS external thread, there is

$$
\begin{aligned}
& h=P-(P / 6)=0.833 \mathrm{P} \\
& b=0.5 P \\
& l=\frac{H}{2}-\frac{H}{6}=0.289 P
\end{aligned}
$$

To bring these values into (4), (5), (6), (7), there are

$$
\left.\begin{array}{l}
\delta_{1 b}=\frac{0.034 F_{n} \cos \alpha}{E_{b}} \\
\delta_{2 b}=\frac{1.08 F_{n} \cos \alpha}{E_{b}} \\
\delta_{3 b}=\frac{0.229 F_{n} \cos \alpha}{E_{b}} \\
\delta_{4 b}=\frac{1.18 F_{n} \cos \alpha}{E_{b}}
\end{array}\right\}
$$

For IOS internal thread, there is

$$
\begin{aligned}
& h=P-(P / 8)=0.875 \mathrm{P} \\
& b=0.5 P \\
& l=\frac{H}{2}-\frac{H}{8}=0.325 P
\end{aligned}
$$

To bring these values into (4), (5), (6), (7), there are

$$
\left.\begin{array}{l}
\delta_{1 n}=\frac{0.073 F_{n} \cos \alpha}{E_{b}} \\
\delta_{2 n}=\frac{1.15 F_{n} \cos \alpha}{E_{b}} \\
\delta_{3 n}=\frac{0.294 F_{n} \cos \alpha}{E_{b}} \\
\delta_{4 n}=\frac{1.14 F_{n} \cos \alpha}{E_{b}}
\end{array}\right\}
$$

The total deformation of the external thread tooth of bolt and the thread tooth of the nut are as follows: 


$$
\begin{gathered}
\delta_{b}=\delta_{1 b}+\delta_{2 b}+\delta_{3 b}+\delta_{4 b}=\frac{q_{(\mathrm{x})} p^{2} \lambda_{b}}{E_{b} \pi d_{2} h} \\
\delta_{n}=\delta_{1 n}+\delta_{2 n}+\delta_{3 n}+\delta_{4 n}=\frac{q_{(\mathrm{x})} p^{2} \lambda_{n}}{E_{n} \pi d_{2} h}
\end{gathered}
$$

Where, $p$-Thread pitch; $d_{2}$-Thread diameter value, $\lambda_{b}, \lambda_{n}$-The dimensionless coefficient of the deformation degree of the thread teeth under the action of axial force, which is mainly related to the thread specification.

\subsection{Load distribution of thread connection}

$\delta_{n}(0)$ and $\delta_{b}(0)$ indicate the deformation of nut and bolt thread at the origin. $\delta_{n}(x)$ and $\delta_{b}(0)$ express the deformation of nut and bolt thread in $X=x . \varepsilon_{n}(x)$ and $\varepsilon_{b}(x)$ represent the amount of compression and elongation of the bolt and nut. With the theory of elastic material, the follows can be obtained.

$$
\begin{aligned}
& \varepsilon_{b}(x)+\varepsilon_{n}(x)=\delta_{b}(x)+\delta_{n}(x)-\delta_{b}(0)-\delta_{n}(0) \\
& \xi=\frac{1}{E_{b} \cdot A_{b}}+\frac{1}{E_{n} \cdot A_{n}}, \quad \gamma=P^{2} \cdot\left(\frac{\lambda_{b}}{E_{b}}+\frac{\lambda_{n}}{E_{n}}\right) \cdot \frac{1}{\pi \cdot d_{2} \cdot h}
\end{aligned}
$$

there is,

$$
\xi \cdot \int_{0}^{x} F(\mathrm{x}) \cdot d x=\gamma \cdot[\mathrm{q}(\mathrm{x})-\mathrm{q}(0)]
$$

After two derivation, the general solution can be solved.

$$
q(\mathrm{x})=\frac{F_{b} \cdot \lambda}{\sinh (\lambda \mathrm{L})} \cdot \cosh (\lambda \mathrm{x})
$$

The formula (14) shows that the axial force of the bolt connection is monotonically increasing in the range of $x=0$ to $x=L$.

There is a relationship between the axial force of the thread tooth and the total axial force.

$$
\frac{F(\mathrm{x})}{F_{b}}=\frac{\sinh (\lambda \mathrm{x})}{\sinh (\lambda L)}
$$

Taking the M36 connecting bolts most used in the TBM cutter as an example, the tensile strength and yield strength of the model are $1030 \mathrm{MPa}$ and $930 \mathrm{MPa}$, the bolt material is $40 \mathrm{Cr}$, and the working load is $370 \mathrm{kN}$. The screw pitch is 4 , with a total of 7 screw threads, the total rotation distance is $28 \mathrm{~mm}$. Through the formula (14) and (15) calculation, the law of the axial force of the thread can be got, and the ratio of the load to the axial load exerted on the shaft ends of each pair of thread teeth. The result is shown in Table 1.

Table 1 The axial load distribution of bolt and the thread force

\begin{tabular}{|c|c|c|c|c|c|c|c|}
\hline $\begin{array}{c}\text { Distance from the connecting } \\
\text { surface of the N tooth }\end{array}$ & 1 & 2 & 3 & 4 & 5 & 6 & 7 \\
\hline $\mathrm{X}(\mathrm{mm})$ & 4 & 8 & 12 & 16 & 20 & 24 & 28 \\
\hline Axial force(kN) & 104 & 173.6 & 227.3 & 271.3 & 308.7 & 341.4 & 370 \\
\hline Local axial force (kN) & 104 & 69.6 & 53.7 & 44 & 37.4 & 32.7 & 27.6 \\
\hline $\begin{array}{c}\text { The percentage of each tooth load in } \\
\text { the total load(\%) }\end{array}$ & 29.1 & 17.8 & 15.4 & 12.1 & 10.9 & 8.3 & 7.5 \\
\hline
\end{tabular}

Considering the relationship (14) and table 1, it is known that the load of thread tooth decreases with the increase of the distance from the bottom. Load of thread tooth at the bottom is $26 \%$ of the 
first thread load, and in the work, the first thread near the bottom is $28.1 \%$ of the axial load applied at the thred end. For example, for the first four thread teeth near the root, the load account for $73.8 \%$ of the total load. Therefore, the stress concentration at the thread connection root is very serious. In the actual work process, serious stress concentration is very easy to cause fatigue, fracture and other issues.

\section{Finite element analysis of load distribution of standard thread connection}

\subsection{The establishment of axisymmetric model}

When the screw angle is small and the bolt is subjected to axial load only, the bolt connection model is simplified as an axisymmetric form. The simplified method has been widely applied in engineering calculation, and its validity and rationality have also been verified. In order to maintain consistency, the M36 bolt in the theoretical calculation above is used as the finite element analysis model. Using the Solidworks to model, the axisymmetric model is built with the $\mathrm{Y}$ axis as a symmetric axis (as shown in Figure 3). The whole model does not take the screw spiral angle into consideration, and imports the SAT file after modularization in the Abaqus. The axial constraint is added to the end face near the bottom of the nut, and the contact relation between surface to surface mode and the friction coefficient is set up on the contact surface separately. The friction coefficient is obtained from the friction coefficient table, and the contact mode is selected as the single side.

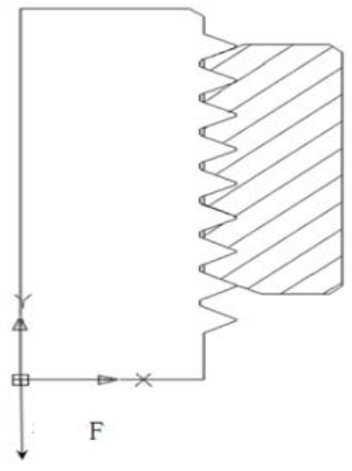

Fig. 3 The axisymmetric model diagram
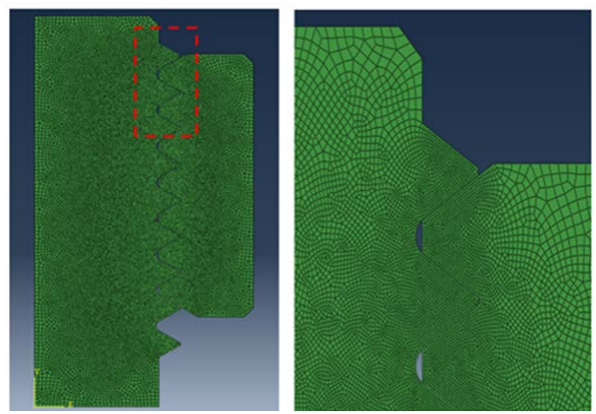

Fig. 4 The grid of thread model

\subsection{Grid partition}

The type of CAX4R linear reduction integral unit is mainly used in large strain analysis. Because of the large stress concentration in the contact area, this type of unit is chosen. In order to reduce the computing time, the dense mesh is in the thread contact area separately, and the loose mesh is in other areas far away from the thread area. The final mesh division results is that the number of internal thread units is 11781 , and the number of nodes is 12087 ,and the number of units in the outer thread area is 24199, and the number of nodes is 24609. The schematic diagram of the grid partition is shown Fig. 4.

\subsection{Contact setting}

The main contact surface is external thread surface and internal thread surface is discretization method of surface to surface. The attribute of contact is set tangential. Friction coefficient is set to 0.15 , and the normal behavior is default setting.

\subsection{Load and constraint setting}

To avoid the phenomenon of stress concentration at the end of the external thread under the action of $370 \mathrm{kN}$ axial force, the type of pressure applied is chosen, and the end face of the external thread is chosen as a fixed constraint surface. 


\subsection{Results analysis}
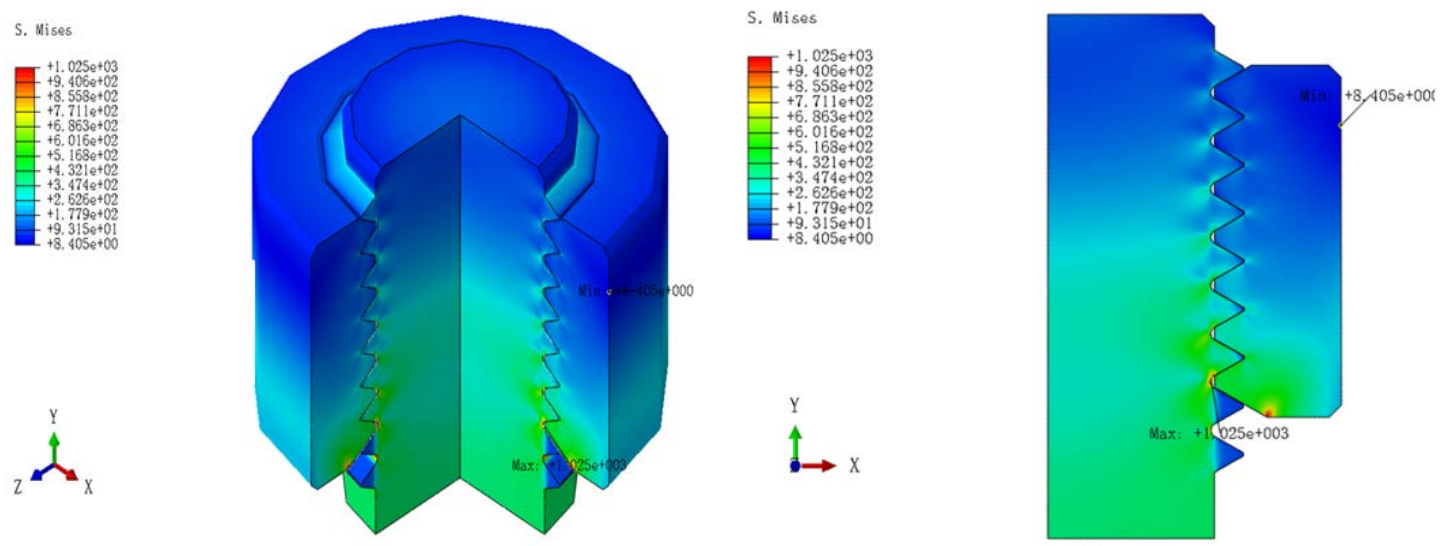

Fig. 5 Three-dimensional equivalent stress nephogram

Figure 5 is the equivalent stress cloud map.It can be known that the first lap near the bottom of thread connection has the maximum stress. And it gradually decreases from the bottom to the top. The severe stress concentration phenomenon appears in the first three laps of the bottom. This is in agreement with the previous theoretical analysis. The axial load of each screw thread is extracted, and the of each thread tooth is obtained, and the axial force distribution of standard screw tooth structure is shown as Table 2.

Table 2 The axial force distribution of standard thread

\begin{tabular}{|c|c|c|c|c|c|c|c|}
\hline $\begin{array}{c}\text { Distance from the connecting surface of } \\
\text { the N tooth }\end{array}$ & 1 & 2 & 3 & 4 & 5 & 6 & 7 \\
\hline Axial load of each screw thread(kN) & 92.9 & 68.8 & 54 & 45.9 & 39.6 & 34 & 33.7 \\
\hline $\begin{array}{c}\text { Percentage of axial load of each circle } \\
\text { thread in total load(\%) }\end{array}$ & 25.1 & 18.6 & 14.6 & 12.4 & 10.7 & 9.2 & 9.1 \\
\hline
\end{tabular}

Known by the Table 2, the first lap near the bottom of thread has the maximum stress, the proportion reached $25.1 \%$, gradually decreases from the bottom to the top of each lap. The severe stress concentration phenomenon appears in the top three laps at the bottom, accounted for 58.3\% of the total, which verified the former theoretical analysis results. Figure 11 is the relationship between the maximum Mises equivalent stress value of the root of each thread tooth and the number of thread teeth connected to the bottom of the distance.

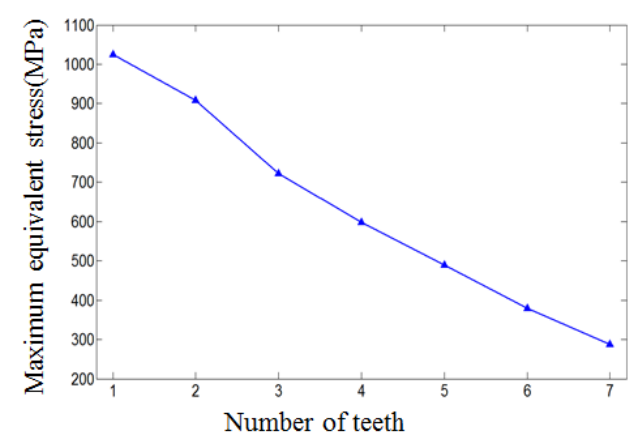

Fig. 6 The maximum Mises equivalent stress distribution about each lap of thread

From Figure 6, it's known that the Mises stress of the first lap thread near root is the largest, reaching $1024 \mathrm{MPa}$, while the Mises stress in the last lap is only $287 \mathrm{MPa}$. The first lap stress is 3.57 times of the last lap stress, and the stress distribution is seriously nonuniform. In order to make the stress distribution uniform, reduce the stress concentration and improve the fatigue life of the connector, the thread connection structure must be improved reasonably. 


\section{Fatigue test of standard thread}

\subsection{Experiment sample}

In order to verify the theoretical analysis and the simulation data, we carried out the test of the standard thread fatigue. The sample material is 304 stainless steel for hot rolling. The bolt structure, as shown in Figure 7, is a double head stud structure of specification M10*1.5, the length of the stud is $100 \mathrm{~mm}$, and the effective thread length at both ends is $30 \mathrm{~mm}$. The effective diameter is $8.7 \mathrm{~mm}$, and the effective area is $58 \mathrm{~mm}^{2}$.

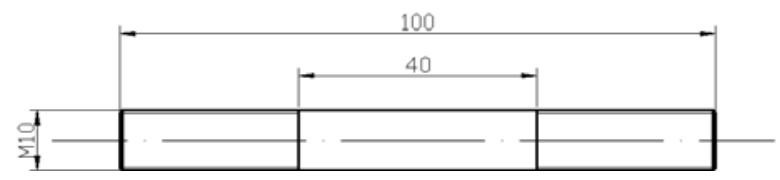

Fig. 7 Double end studs

\subsection{Experimental fixture}

Refer to GB/T26077-2010, 《Axial constant amplitude low cycle fatigue test method》 (GB/T15248-94) and 《Metal axial fatigue test method》(GB3075-82) and other national standards and related equipment requirements, the corresponding parameters of the special fixture designed are as follows.
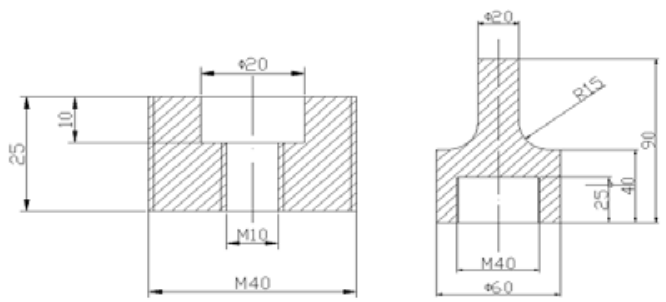

Fig. 8 The experimental fixture

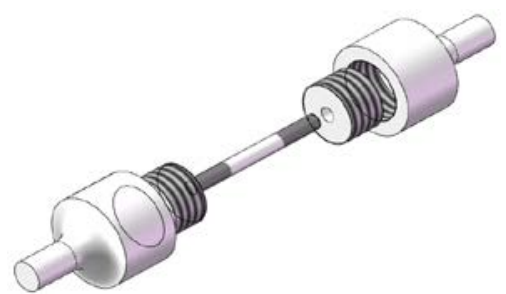

Fig. 9 Samples and fixture installation rendering

\subsection{Experimental equipment}

This experiment uses low frequency hydraulic servo fatigue tensile test machine (model MTSLandmark 370.25) ,as shown in Figure 10. The effective tensile force is $250 \mathrm{kN}$, and the effective dynamic working range is $150 \mathrm{~mm}$. According to the calculation, the working requirements of the tensile test are achieved.

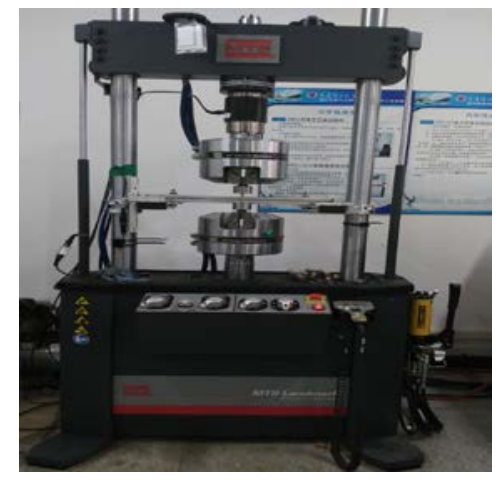

Fig. 10 The MTS fatigue tensile testing machine

\subsection{Experimental parameters}

Loading mode: axial loading. Experimental frequency: 12Hz. Experimental temperature: room temperature. Stress cycling ratio $R$ is 0.1 . Dynamic loading mean is $19.25 k N$. Dynamic loading amplitude is $15.75 \mathrm{kN}$. 


\subsection{Results analysis}

Table 3 is the experimental results recorded, in which the number of cycles eliminated is significantly less than that of samples 1-2 and invalid data samples that the unbroken number 1-6 bolts wihle the number of cycles is over 105. This experiment only verifies the theoretical and simulation results in the previous article, so it does not do the thorough analysis of the fatigue life of the experimental data.

Table. 3 The fracture information of M10 double-headed stud

\begin{tabular}{|c|c|c|c|c|c|c|c|c|c|}
\hline \multirow{2}{*}{ Number } & \multicolumn{7}{|c|}{ 304 stainless steel double head stud } \\
\cline { 2 - 8 } & $1-1$ & $1-2$ & $1-3$ & $1-4$ & $1-5$ & $1-6$ & $1-7$ & $1-8$ \\
\hline $\begin{array}{c}\text { Number of } \\
\text { cycles }\end{array}$ & 37434 & 19512 & 41602 & 39314 & 37768 & 121334 & 39685 & 40402 \\
\hline $\begin{array}{c}\text { Fracture } \\
\text { position }\end{array}$ & $\begin{array}{c}\text { First } \\
\text { tooth }\end{array}$ & $\begin{array}{c}\text { First } \\
\text { tooth }\end{array}$ & $\begin{array}{c}\text { First } \\
\text { tooth }\end{array}$ & $\begin{array}{c}\text { First } \\
\text { tooth }\end{array}$ & $\begin{array}{c}\text { First } \\
\text { tooth }\end{array}$ & Unfractured & $\begin{array}{c}\text { First } \\
\text { tooth }\end{array}$ & $\begin{array}{c}\text { First } \\
\text { tooth }\end{array}$ \\
\hline
\end{tabular}

Figure 11 is a fracture position of a double head stud. It can be seen that the fracture locations of the experimental samples all occur in the root of the first thread of the threaded screwing segment, because the fracture of the selected experimental specimen has no obvious machining defects, so the specimen is all fatigue fracture. This indicates that the axial load of the first thread tooth near the root is the largest in thread connection, and the stress concentration phenomenon is obvious at the root of the screw thread. This experimental result is consistent with the theoretical conclusions deduced above, and proves the correctness of the finite element simulation results.
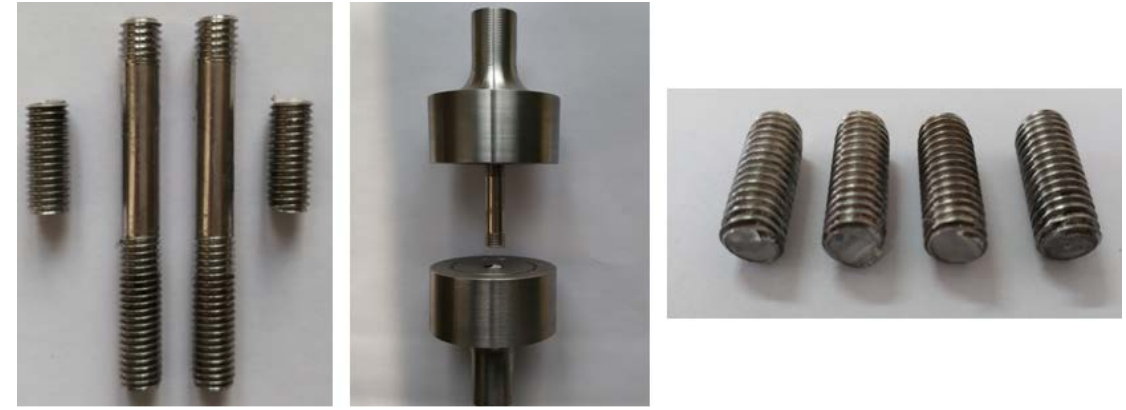
1)1\#、3\# stud
2) overall fracture
3) threaded teeth fracture surface

Fig. 11 Double end studs fracture location

\section{Summary}

On the basis of previous scholars' theory, this paper makes a theoretical calculation and analysis on the law of the load distribution of the thread in the standard thread connection. According to the derived formula, the axial load near the root first circle is the largest, which accounts for $28.1 \%$ of the total axial load, and the axial load from the screw contact surface to the top nut of the nut decreases. Then through the Abaqus finite element software, the thread connection model is analyzed and calculated. The simulation results are similar to the theoretical calculations. The axial load of the first circle near the root is the largest, which accounts for $25.1 \%$ of the total axial load, and the stress concentration appears in the first circle of the thread. Finally, the fatigue tensile test of the thread was designed. The experimental results showed that the fracture position of the bolt occurred in the first lap of the thread under the axial tension. The experimental results are all in agreement with the theoretical calculation and the simulation results. Through a series of analysis and experiments, this paper obtains the distribution law of the load of thread connection, which can provide theoretical guidance for the design of the thread connection. 


\section{Acknowledgements}

This work is supported by the National Natural Science Foundation of China (Grant No.51375001), Major projects of Liaoning science and technology program (2015106016), Special topic for the basic scientific research business of the Central University (DUT16QY11).

\section{References}

[1] Xia Wei-ming, Zheng Xiang, Yang Xiao-jun. FEM simulation of blind bolt connection based on ANSYS [J]. Machinery Design \& Manufacture, 2009, (7):42-44.

[2] Xie Li-yang, Xu hao. Combined mechanism method and fatigue life estimation of bolt stress analysis [J]. Machine Design,1986, 04:26-31.

[3] Shi xiu-yong, Li Guo-xiang. Study on Strength Check of High Intensity Cylinder Head Bolt of Diesel Engine [J]. Diesel Engine, 2006, 28(3):67-75.

[4] Shi xiu-yong, Li Guo-xiang. Three dimensional finite element analysis of engine flywheel bolts[J]. China Mechanical Engineering, 2006,17(8): 845-848.

[5] Fang Dong, Chen Jizhi. Finite element analysis of stress concentration in the root of high strength bolt [J]. Material development and Application, 2007,22(2):37-39.

[6] Sun Yujuan, Liao Ridong, Zhang Weizheng. Study on axial load and stress distribution of threaded joins[J]. Ship Engineering, 2007. (2),26-31.

[7] Kim J, Yoon J C, Kang B S. Finite element analysis and modeling of structure with bolted joints[J]. Applied Mathematical Modelling, 2007, 31(5): 895-911.

[8] Akira Yamamoto, Guo Keqian. Theory and calculation of threaded connection [M]. Shanghai: Shanghai science and Technology Literature Press.1982. 